\title{
Summary of the CKM2016 working group on semileptonic and leptonic $B$ decays
}

\author{
Brian Hamilton* \\ University of Maryland, USA \\ E-mail: brian.hamiltonecern.ch
}

\section{Aoife Bharucha}

CPT, UMR7332, CNRS and Aix-Marseille Université and Université de Toulon 13288 Marseille,

France

E-mail: aoife.bharuchaecpt.univ-mrs.fr

\section{Florian Bernlochner}

University of Bonn, Germany

E-mail: florian.bernlochnerecern.ch

Semileptonic and leptonic decays of beauty hadrons provide important input to, and constraints on, the CKM unitarity triangle. In addition to standard model parameters, these can also probe the parameter space of new physics models which induce charged current transitions. In this summary, we present an overview of new work in this field as presented at the CKM2016 conference at TIFR in Mumbai, India. Topics include measurement of CKM elements and theoretical issues in their extraction, as well as the emerging work on new physics searches in the semileptonic decays to tau leptons from both experimental and theoretical perspectives.

9th International Workshop on the CKM Unitarity Triangle

28 November - 3 December 2016

Tata Institute for Fundamental Research (TIFR), Mumbai, India

${ }^{*}$ Speaker. 


\section{Introduction}

Semileptonic decays of the form $b \rightarrow q \ell \bar{v}$ and the closely related leptonic decays $B^{-} \rightarrow \ell \bar{v}$ are among the simplest charged-current transitions in the standard model. They are dominated by treelevel graphs involving the emission of a virtual $W$ boson with a single CKM factor, and interactions between the leptonic and hadronic parts of the final state lack the kinds of nonperturbative effects present in hadronic $B$ decays. These properties mean that semileptonic decays provide an excellent probe of $b \rightarrow c$ and $b \rightarrow u$ charged current interactions, provided that an adequate description can be provided for the matrix elements governing the hadron level $H_{b} \rightarrow H_{q}+X$ transitions. They are thus very important for providing information on the CKM elements $\left|V_{c b}\right|$ and $\left|V_{u b}\right|$, and constraining the length the side of the unitarity triangle opposite $\beta / \phi_{1}$. At the same time, this simple decay structure allows for their use as a probe for new interactions, particularly in the form of tests of lepton flavor universality, which demands that the amplitudes for the semileptonic decays to $e, \mu$, and $\tau$ leptons be equal up to explicitly mass-dependent effects.

In the two years since the last CKM workshop, some of the key results were:

- Exclusive $V_{u b}$ : The calculation of the form factors for $B \rightarrow V$ in LCSR, and for $\Lambda_{b} \rightarrow p$ and $B_{s} \rightarrow K$ in Lattice QCD [1]. There have further been experimental updates to $B \rightarrow \pi$ (resulting in a precise determination of $V_{u b}$ from a combination by HFAG [2]) and $B \rightarrow V$, and a measurement of $\Lambda_{b} \rightarrow p \ell v$ at LHCb.

- Inclusive $V_{u b}$ : The development of neural networks for use in analyzing inclusive semileptonic B decays: NNVub [3], as well as new Babar result probing the endpoint region [4].

- Exclusive $V_{c b}$ : The update of $B \rightarrow D^{*} \ell v$ [5] and $B \rightarrow D \ell v$ [6] from the full Belle $\Upsilon(4 S)$ dataset as well as the calculation on the Lattice of the form factors for $B_{S} \rightarrow D_{s}$ [7].

- Inclusive $V_{c b}$ : An updated HFAG result for 2016 was presented [2], and improvements to the theory calculation using the GGOU method at $\mathscr{O}\left(\alpha_{s} \mu_{\pi / G}^{2} / m_{b}^{2}\right)[8]$.

- Leptonic decays: In 2015 a semileptonic tag result for $B \rightarrow \tau v$ was released by Belle [9]

- $R_{D}$ and $R_{D}^{*}$ : The HFAG combination of $R_{D^{*}}$ and $R_{D}$ including correlations shows a deviation of $\sim 4 \sigma$ from the Standard Model [2]. The 2015 LHCb measurement of $R_{D^{*}}$ has similar central value to 2012/13 BaBar [10]. There are also three new measurements from Belle for $R(D)[11]$ and $R_{D^{*}}[11,12,13]$, two of which with leptonic taus.

In the following we will discuss these results in more detail, providing a brief overview of the recent progress in the field of semi-leptonic $B$ decays both on the experimental and theoretical side.

\section{Exclusive decays}

\subsection{Exclusive $b \rightarrow c \ell \bar{v}_{\ell}$}

The discussion on exclusive $b \rightarrow c \ell \bar{v}_{\ell}$ was dominated by two new measurements by the Belle collaboration and the applicability of the CLN parametrization [14]. Growing indications seem to imply that at least a partial explanation for the difference between $\left|V_{c b}\right|$ from inclusive and exclusive 
determinations could be due to underestimated theory uncertainties on the sum-rule assumptions incorporated in Ref. [14]. The state-of-the-art determination of $\left|V_{c b}\right|$ for $B \rightarrow D \ell \bar{v}_{\ell}$ combines the experimentally measured distributions with beyond zero-recoil lattice information $[6,15]$ and carries out the extraction in a combined theory-data fit. For $B \rightarrow D^{*} \ell \bar{v}_{\ell}$ no lattice information beyond zero-recoil [16] is available yet, but providing this is seen as a priority by the lattice community. Precision measurements of $B \rightarrow D \ell \bar{v}_{\ell}$ and $B \rightarrow D^{*} \ell \bar{v}_{\ell}$ not only provide an avenue for $\left|V_{c b}\right|$, but also play a crucial role in predicting the relative rate of decays to the tau lepton relative to light leptons. Belle showed two recent measurements that provide fully unfolded decay distributions for the recoil parameter $w=v_{B} v_{D^{(*)}}$ for $B \rightarrow D \ell \bar{v}_{\ell}$ and $B \rightarrow D^{*} \ell \bar{v}_{\ell}$ decays and decay angles for $B \rightarrow D^{*} \ell \bar{v}_{\ell}$. An example of the measured spectra is shown in Figure 1, taken from Refs. [17, 5]. Fitting the $B \rightarrow D \ell \bar{v}_{\ell}$ data using CLN or the model independent BGL [18] parametrization results in

$$
\left|V_{c b}\right|=(39.9 \pm 1.3) \times 10^{-3}, \quad\left|V_{c b}\right|=(40.8 \pm 1.1) \times 10^{-3},
$$

respectively. The value recovered from the BGL fit is noticeably compatible with inclusive determinations of $\left|V_{c b}\right|$, reducing a long standing tension. A more detailed summary may be found in Ref. [19]. Fitting the $B \rightarrow D^{*} \ell \bar{v}_{\ell}$ data with CLN Belle reports

$$
\left|V_{c b}\right|=(37.4 \pm 1.3) \times 10^{-3} \text {. }
$$

Belle also reported unfolded decay distributions for $B \rightarrow D^{*} \ell \bar{v}_{\ell}$ for the first time. These results were used in more recent publications [20,21,22] to explore form factor parameterizations beyond CLN and report a sizeable shift when using BGL [18] of

$$
\left|V_{c b}\right|=(41.7 \pm 1.7) \times 10^{-3},
$$

a value very compatible with the inclusive determinations of $\left|V_{c b}\right|$. Finally there are recent efforts to extract $\left|V_{c b}\right|$ from $B_{s}$ [23] and $B_{c}$ decays, relevant progress on the Lattice can be found in Refs. [15, 24] respectively.
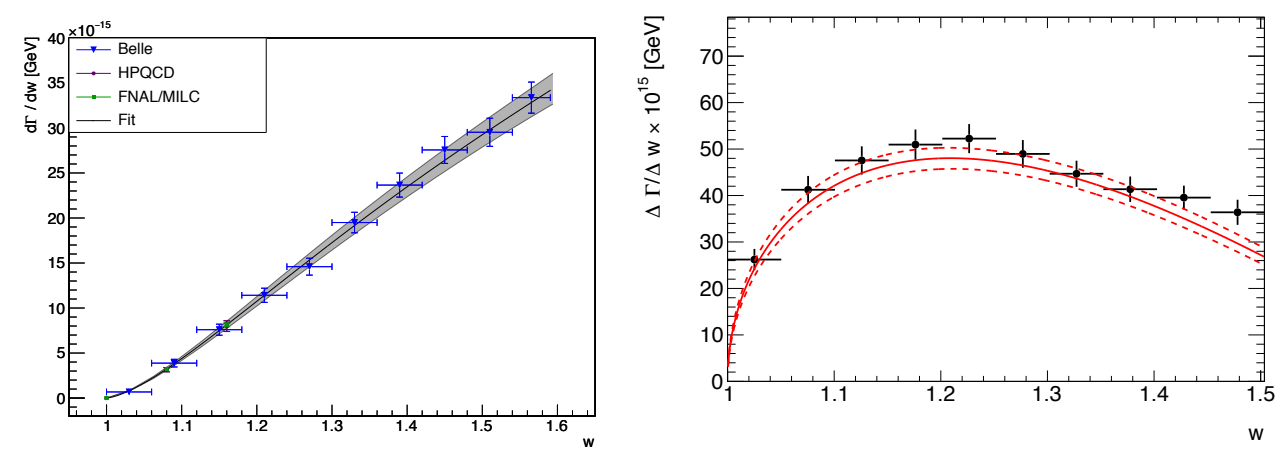

Figure 1: The measured recoil parameter spectra from (left) $B \rightarrow D \ell \bar{v}_{\ell}$ and (right) $B \rightarrow D^{*} \ell \bar{v}_{\ell}$ from Refs. [17, 5]. 


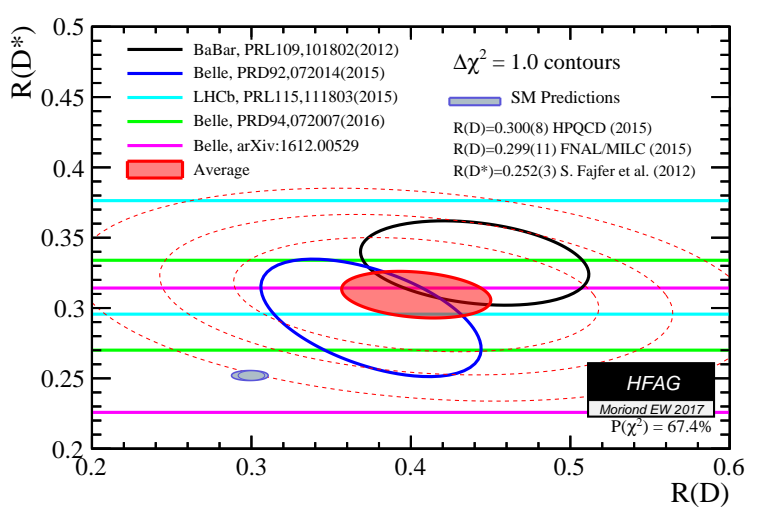

Figure 2: Current measurements of $R(D)$ and $R\left(D^{*}\right)$ with average and correlations. Taken from Ref. [2].

\subsection{Exclusive $b \rightarrow c \tau \bar{v}_{\tau}$ and tests of Lepton Flavour Universality}

In the past few years, much experimental and theoretical effort has been devoted to understanding the hints of a possible excess of $\bar{B} \rightarrow D^{(*)} \tau \bar{v}$ decays relative to $\bar{B} \rightarrow D^{(*)} \ell \bar{v}, \ell=\mu$ or $e$, predicted to be $[1,25]$

$$
\begin{gathered}
R(D) \equiv \frac{\mathscr{B}(\bar{B} \rightarrow D \tau \bar{v})}{\mathscr{B}(\bar{B} \rightarrow D \ell \bar{v})}=0.300 \pm 0.008 \\
R\left(D^{*}\right) \equiv \frac{\mathscr{B}\left(\bar{B} \rightarrow D^{*} \tau \bar{v}\right)}{\mathscr{B}\left(\bar{B} \rightarrow D^{*} \ell \bar{v}\right)}=0.252 \pm 0.003
\end{gathered}
$$

The $R(D)$ prediction has been updated and improved over the past two years with lattice form factor fits from both the HPQCD [6] and FNAL/MILC [26] collaborations. Both calculations use $2+1$ flavor improved sea quark actions from MILC and probe the form factors across the full $q^{2}$ range of the decay. The former uses NRQCD bottom quarks with HISQ valence and charm quarks, while the latter makes use of improved Fermilab-interpretation Wilson fermions for bottom and charm and asqtad-improved staggered light valence quarks. The results are in excellent agreement, and a campaign is underway to extend these kinds of lattice calculations to the $D^{*}$ final states as well as other $B$ hadron flavors.

The earlier BaBar result [27] using the hadronic tagging technique on the full dataset has been joined by three new results using the full Belle dataset. The first of these uses a hadronic $B_{\text {tag }}$ and measures both $R(D)$ and $R\left(D^{*}\right)$ by simultaneously fitting the missing mass peak at zero and a neural net output at high missing mass for both the $D$ and $D^{*}$ dominated samples [11]. They find a result consistent with both the Standard Model and the $2012 \mathrm{BaBar}$ result, $R(D)=0.375 \pm 0.064 \pm 0.026$ and $R\left(D^{*}\right)=0.293 \pm 0.038 \pm 0.015$. In addition, Belle has employed a semileptonic $B_{\text {tag }}$ technique to provide an independent measurement of $R\left(D^{*}\right)$ alone, employing the unassociated extra calorimeter energy along with a multivariate classifier based on the event kinematics to discriminate the various contributions. This result is in agreement with the hadronically-tagged result, yielding $R\left(D^{*}\right)=0.302 \pm 0.030 \pm 0.011$. Finally, Belle has also presented a preliminary new result using a hadronic $B_{\text {tag }}$, but analyzing the $\tau^{-} \rightarrow \pi^{-}\left(\pi^{0}\right) \nu$ decay channel. This approach sacrifices 
the identical reconstruction of the signal and normalization decays in the leptonic tau decay channel, but in exchange gains experimental sensitivity to the $\tau$ decay angles and polarization. Belle quotes a preliminary result of $R\left(D^{*}\right)=0.270 \pm 0.035_{-0.025}^{+0.028}$ and $P_{\tau}=-0.38 \pm 0.51_{-0.16}^{+0.21}$ [13].

A new contributor in this area is LHCb, who in 2015 published their own measurement of $R\left(D^{*}\right)$ using the 2011 and 2012 collision data at the Large Hadron Collider. They use a technique involving a controlled but imprecise approximation of the total $B$ momentum to estimate the missing mass, $q^{2}$ and lepton energy and perform a 3-d fit for the signal. The lack of a full-event reconstruction results in higher backgrounds from other $B$ decays, in particular those of the form $\bar{B} \rightarrow D^{*} D X$, which can only be partially suppressed by use of charged-track isolation. Still, the large statistics proves to be quite powerful and they obtain a similar sensitivity to the Belle results, reporting $R\left(D^{*}\right)=0.336 \pm 0.027 \pm 0.030$ [10]. Though the quoted systematic uncertainty is larger than the statistical uncertainty, it is dominated by a combination of control sample statistics and finite simulation size, and so does not constitute a limit on future sensitivity. They expect results in the near future using the same dataset for a combined and improved $R(D)$ vs $R\left(D^{*}\right)$ measurement, as well as a measurement using the $\tau^{-} \rightarrow \pi^{-} \pi^{+} \pi^{-}\left(\pi^{0}\right) \nu$ decay. The latter is a particularly attractive mode at $\mathrm{LHCb}$ due to the very large lab momentum involved: imposing strict requirements on the spatial order of the reconstructed $B, D$ and $\tau$ decay vertices allows for the suppression of the largest backgrounds.

The current experimental situation is summarized in Figure 2 [2]. The $p$-value of the world average with respect to present SM calculations is $8.8 \times 10^{-5}(\approx 3.9 \sigma)$. Though this does not rise to the level of a definitive observation of a deviation from the SM, the measurements of the past two years have strengthened rather than relaxed the tension from [27] and so there has been much theoretical effort to understand what sorts of New Physics models could generate an enhancement of this size while remaining consistent with all other available data.

The large relative enhancement in $R\left(D^{*}\right)$ disfavors a charged Higgs of the type-II two Higgs double model, though a MSSM-like type-III two Higgs doublet structure with flavor violation in the up sector could accommodate both. Leptoquark explanations were also presented to account for the possible enhancement $[29,30,31,32]$, with certain models generating both an $R\left(D^{(*)}\right)$ enhancement as well as deviations from lepton universality in $b \rightarrow s \ell^{+} \ell^{-}$processes which may be dependent on the spin of the hadronic final state. More exotic models have also been put forward including the introduction of a scale at which spacetime becomes non-commutative (using the same framework as, e.g. [33]). A consensus has emerged on the theoretical front that differential distributions must be utilized to distinguish NP models and more clearly observe non-SM-like effects in these decays $[34,29]$. The possibility of investigating such observables with the present data is a topic of active discussion, but will be challenging with the present small signal yields $(O(100))$ at the B-factories or the large backgrounds and poorly-constrained kinematics at LHCb.

\subsection{Exclusive $b \rightarrow u \ell \bar{v}_{\ell}$}

The three most recent LCSR calculations were carried out in 2012 (2-loop corrections to $f_{+}(0)$ for $B \rightarrow \pi$ [35]), 2014 (Bayesian analysis also $f_{+}\left(q^{2}\right)$ for $B \rightarrow \pi$ at NLO [36]) and 2015 (update to the form factors for $B \rightarrow V$ from LCSR [37]). For $B \rightarrow \pi$, a NNLO $\left(\mathscr{O}\left(\alpha_{s}^{2} \beta_{0}\right)\right)$ calculation of $f_{+}(0)$ was performed, with the result $f_{+}(0)=\left(0.262_{-0.023}^{+0.020}\right)$ with uncertainties $\lesssim 9 \%$ [35]. This calculation tested the argument that radiative corrections to $f_{+} f_{B}$ and $f_{B}$ should cancel when both 
calculated in sum rules (the 2-loop contribution to $f_{B}$ in QCDSR is sizeable). More recently unitarity bounds and extrapolation were used to perform a Bayesian analysis of the form factor $f_{+}\left(q^{2}\right)$ for $B \rightarrow \pi$ [36]. Prior distributions were taken for inputs, a likelihood function was constructed based on fulfilling the sum rule for $m_{B}$ to $1 \%$, and posterior distributions were obtained using Bayes' theorem. The posterior distributions of the inputs differed only for $s_{0}$, which was pushed to higher values $s_{0}=41 \pm 4 \mathrm{GeV}$ (mainly due to the choice of $m_{b}$ ). Finally the results were fit to the BCL parameterization, finding a central value of $f_{+}(0)=0.31 \pm 0.02$ (larger due to the central values of $m_{b}$ and $\mu$ ). Obtaining $f_{+}\left(q^{2}\right)$ and the first two derivatives at 0 and $10 \mathrm{GeV}^{2}$ has allowed the extrapolation to higher $q^{2}$ using improved unitarity bounds.

The latest updates to $V_{u b}$ from LCSR come from the channels $B \rightarrow \rho / \omega$ : updated LCSR results including correlations and employing the eqution of motion to constrain the sum rules parameters are now available [37]. The result for $\left|V_{u b}\right|$ from $B \rightarrow \rho \ell v$ has comparable errors to the $B \rightarrow \pi$ determination. In general the $B \rightarrow V$ results agree with previous exclusive determinations and global fits within errors.

At LHCb, $20 \%$ of b-hadrons produced are $\Lambda_{b}$ baryons, and smaller backgrounds of $p$ in the underlying event make a determination of $\left|V_{u b}\right|$ in this system attractive. By measuring the ratio of branching fractions of $\Lambda_{b} \rightarrow p \ell v$ to $\Lambda_{b} \rightarrow \Lambda_{c} \ell v$ [38], LHCb has extracted $\left|V_{u b}\right| /\left|V_{c b}\right|$ using Lattice QCD results for the form factors [39]. The isolation of the proton-muon vertex is important to reduce (but not eliminate) backgrounds; for this reason a multivariate classifier is used. A fit is made in the corrected mass (approximately the transverse mass with respect to the vertex displacement direction) which peaks at the true $\Lambda_{b}$ mass. The effects of a twofold ambiguity in the full momentum of $\Lambda_{b}$ is reduced by requiring both solutions to be above $15 \mathrm{GeV}^{2}$. The error is presently systematics dominated, mostly due to the uncertainty on the branching fraction of $\Lambda_{c} \rightarrow p K \pi$, as well as the trigger and tracking efficiency. A future target for LHCb is the channel $B_{s} \rightarrow K \mu \nu$. Again, the form factors from lattice are available with good accuracy [7]. While there is a large production of $B_{s}$ (14\% of all b-hadrons), the backgrounds are harder here as there are many more sources of $\mu+K$ than $\mu+p$, the most dangerous being the $B_{s} \rightarrow K^{*} \mu \nu$ which has to be fitted along with the signal. Possible methods to increase sensitivity to this channel were discussed.

A comprehensive overview of results from the $B$ factories was presented, of which the highlight was the combination of $B \rightarrow \pi \ell v$ measurements, where the most precise measurements (i.e. Refs. [40, 41, 42, 43]) were averaged with a likelihood fit, resulting in a very precise value of $\left|V_{u b}\right|^{\pi \ell v}=\left(3.65 \pm 0.09_{\exp } \pm 0.11_{\text {theo }}\right) \times 10^{-3}$, a $4 \%$ relative uncertainty [2], using a BCL fit, the LCSR NNLO result for $f_{+}(0)$ [35] mentioned above and the Lattice results from Ref. [44]. Updates to $B \rightarrow\left(\rho, \omega, \eta^{(\prime)}\right) \ell v$ measurements $[41,42,43]$ were also presented, in particular an untagged result from BaBar and a tagged result from Belle for $B \rightarrow \rho \ell v$, resulting in $\left|V_{u b}\right|^{\rho \ell v}=$ $\left(3.21 \pm 0.11_{\exp } \pm 0.22_{\text {theo }}\right) \times 10^{-3}$ using the LCSR form factor calculation discussed earlier [37] The expected $\left|V_{u b}\right|^{\pi \ell v}$ precision with Belle II dataset and LQCD forecasts are $1.7 \%$ and $1.3 \%$ for tagged and untagged analyses respectively, from a Monte-Carlo study (provided by the Belle II Theory Interface Platform, a detailed report is in preparation) with Lattice QCD forecasts (thanks to A. Kronfeld, T. Kaneko and S. Simula). The projections for the other channels are not yet available, however with the expected sample sizes it should be possible to perform a full angular analysis, such that progress on the theory side would be desirable. 


\section{Inclusive decays}

\subsection{Inclusive $b \rightarrow c \ell \bar{v}_{\ell}$}

The determination of inclusive $\left|V_{c b}\right|$ is based on using the heavy-quark expansion and the combined analysis of measured moments of the decay spectra characterizing the $b \rightarrow c \ell \bar{v}_{\ell}$ transition. The moments of the lepton spectrum and the invariant mass squared spectrum can be related to five non-perturbative parameters in $b \rightarrow c \ell \bar{v}_{\ell}$ and determined using a $\chi^{2}$ fit. Predicting the moments requires knowledge of $m_{c, b}$, and the two most important sources of theory uncertainties are from missing higher order corrections in the heavy-quark expansion and terms that violate quark-hadron duality. These uncertainty sources are added into the fit by constructing a covariance matrix that can be used in the extraction. An example for the fitted first lepton moment and the partial inclusive $b \rightarrow c \ell \bar{v}_{\ell}$ branching fraction are shown in Figure 3. The shown fit uses the kinetic scheme [2] and finds

$$
\left|V_{c b}\right|=(42.19 \pm 0.79) \times 10^{-3}, \quad m_{b}^{\text {kin }}=4.554 \pm 0.018, \quad \mu_{\pi}^{2}=0.464 \pm 0.076 .
$$

This fit includes recent sum-rule results on the charm-quark mass and $\mathscr{O}\left(\alpha_{s} \Lambda_{Q C D}^{2} / m_{b}^{2}\right)$ contributions. The error on $\left|V_{c b}\right|$ is dominated by theory uncertainties of the measured moments. The fitted $\chi^{2}$ is 14.6 for 43 degrees of freedom. Without the inclusion of theory uncertainties the fit has a p-value of below 5\%, which illustrates their importance.

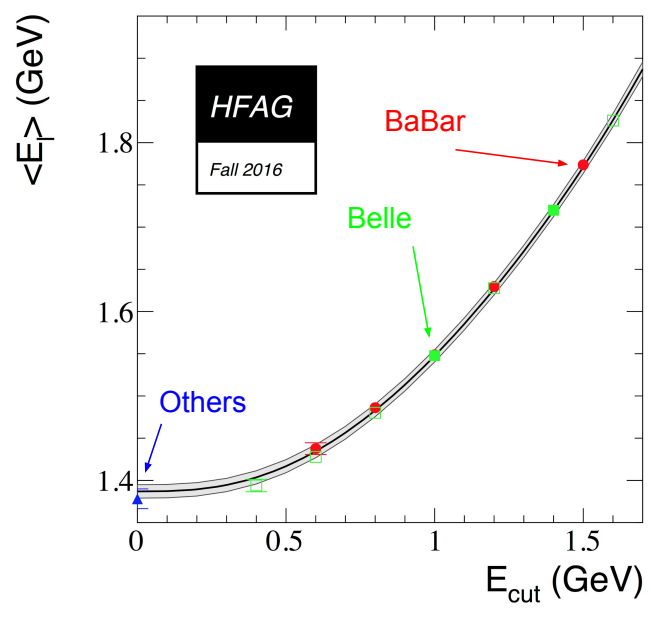

First Lepton Moment

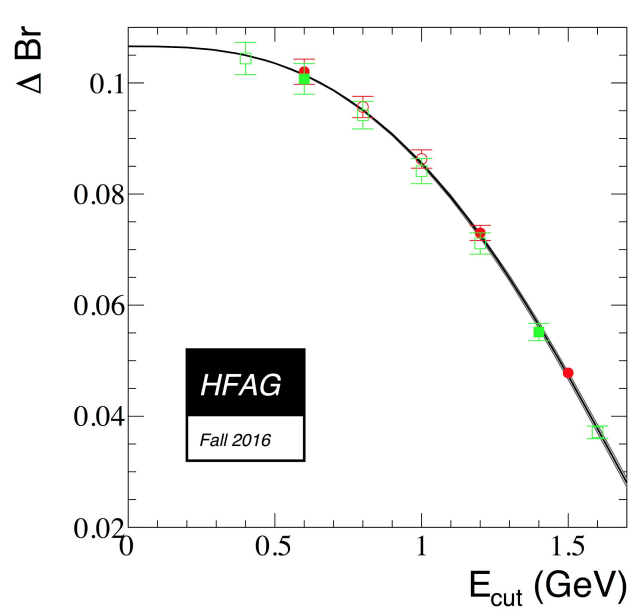

Partial BF (zeroth order moments)

Figure 3: The first moment of the inclusive lepton momentum and the partial $b \rightarrow c \ell \bar{v}_{\ell}$ branching fraction are shown. Both plots are from Ref [2].

On the theory side, recent progress was reported on including corrections up to $\mathscr{O}\left(1 / \mathrm{m}_{b}^{4,5}\right)$. Such corrections have been incorporated by [45] leading to a modest shift of

$$
\left|V_{c b}\right|=(42.00 \pm 0.64) \times 10^{-3},
$$

leaving the inclusion of $\mathscr{O}\left(\alpha_{s} / m_{b}^{3}\right)$ and $\mathscr{O}\left(\alpha_{s}^{3}\right)$ effects as the next thing to include to further reduce the theory error. 


\subsection{Inclusive $b \rightarrow u \ell \bar{v}_{\ell}$}

The kinematic region for $b \rightarrow u$ inclusive is quite resrticted by experimental and theoretical constraints, i.e. due to cuts designed to reduce sensitivity to non-perturbative effects, and to avoid large backgrounds from $b \rightarrow c$. Despite the tension with exclusive $\left|V_{u b}\right|$, results from different calculations (BLNP [46], GGOU [47] and DGE [48]) and acceptance regions, inclusive $\left|V_{u b}\right|$ appears internally consistent. Furthermore the tension between the exclusive and inclusive results for $\left|V_{u b}\right|$ may, in fact, be receding due to new Lattice QCD results and a new Babar endpoint analysis. Additional progress in this direction is expected with Belle-II and LHCb data in a number of channels.

Inclusive observables may be expressed using the OPE as a double series in $\Lambda / m_{b}$ and $\alpha_{s}$ in terms of non-perturbative parameters, which for $\left|V_{u b}\right|$ are $m_{b / u}, \mu_{\pi / G}^{2}$ and $\rho_{D, L S}^{3}$. The dominant nonperturbative contributions are usually resummed into a shape function $f\left(k_{+}\right)$. This shape function is accessed using constraints from the OPE and fits to semi-leptonic data. These shape functions depend on $q^{2}$, and may be written in terms of simple 2 parameter functional forms. The use of artificial neural networks, via the tool NNVub, to parameterize shape functions without bias and extract $\left|V_{u b}\right|$ using the GGOU method from theoretical constraints and data, together with HQE parameters, in a model independent way (without assumptions on functional form) has recently been developed [3]. This would provide a means of estimating the uncertainty arising from shape functions in the $B \rightarrow X_{u} \ell v$ channel. The method can easily be extended to include new information, i.e. Belle-II experimental data or OPE constraints, by reweighting (instant) or retraining (slow). Finally, the comparison with data will validate the inclusive approach to Vub in a much more stringent way, and in the future it is hoped that NNVub and similar tools will be able to reduce the uncertainty on inclusive $\left|V_{u b}\right|$ to the level of that on inclusive $V_{c b}$.

This idea is conceptually similar to the SIMBA approach presented at earlier instalments of this conference (see e.g. Ref. [49]), where the shape function is modelled in a model independent way using an orthogonal set of basis functions; in the shown framework the leading shape function from $b \rightarrow u$ can be related to the leading shape function of $b \rightarrow s \gamma$ and to moment constraints from $b \rightarrow c$ transitions. This allows one to measure inclusive $\left|V_{u b}\right|$ in the experimentally most precise region, where $b \rightarrow c$ is forbidden and shape function effects are large. Such an approach seems a promising way to utilize Belle II data.

Obtaining $\left|V_{q b}\right|$ from inclusive channels relies on dimension seven and eight HQET operators. A general approach to constructing HQET operators has been devised, using tensor decomposition and certain symmetries (e.g. PT symmetry and hermiticity) of the final HQET matrix elements to systematically write the operators in a given dimension. This method has a number of applications: different bases can easily be related; higher dimension operators can be constructed i.e. dimension 9; moments of the leading order shape function up to and including dimension 9 HQET operators can be obtained; it can be verified that the set of operators is complete; the HQET operators may be related to NRQCD operators [50]. As a result of the last relationship, it is postulated that the structure of effective field theories may be simpler than was previously thought. As mentioned earlier, a new BaBar result has recently been published involving an inclusive fit to $e^{ \pm}$spectrum [4]. The fit was carried out extending in the range $\left[p_{\min }, 2.7\right] \mathrm{GeV}$, where $p_{\min }$ is from 0.8 to $2.1 \mathrm{GeV}$, avoiding details of the endpoint (shape function dominated) region by use of a single wide bin 
there. A Hybrid fit model incorporates exclusive final states into the inclusive spectrum. The total experimental error on $\left|V_{u b}\right|$ is below $4 \%$ for $p_{\min }<2.4 \mathrm{GeV}$.

In addition, several possible improvements using the large Belle II dataset should be mentioned; first a detailed composition of the $B \rightarrow X_{c} e v$ background requiring measurement of exclusive final states e.g. $D^{(*)} n \pi, D^{(*)} \eta$. The modelling of $B \rightarrow X_{u} \ell v$ decays incorporating resonant and non-resonant states into the Monte-Carlo can be improved by measuring exclusive e.g. $B \rightarrow n \pi e v$ final states.

\section{Leptonic $B$ decays}

Closely related to the semileptonic $B$ decays are the purely leptonic decays $B^{-} \rightarrow \ell \bar{v}$. As in the semileptonic decays, these are tree-level decays but in an annihilation topology. These provide a potentially powerful tool to measure the CKM element $\left|V_{u b}\right|$, as they involve only a single nonperturbative parameter, the $B$ meson decay constant $f_{B}$. In combination with a measured value of $\left|V_{u b}\right|$, such decays also can be used to search for new physics contributions. The challenge then is to cleanly measure the absolute decay rate in these rare modes that have substantial missing energy.

Progress in leptonic decays has been slow in the gap between the end of $B$-factory running and the start of the Belle II physics program. Belle has supplemented their hadronically-tagged $B^{+} \rightarrow$ $\tau^{+} v$ analysis [51] with a new result using semileptonic tags with similar statistical uncertainty but larger systematic uncertainty [9]. The preferred value of $\left|V_{u b}\right|$ from the $B^{+} \rightarrow \tau^{+} v$ is presently in much better agreement with $\sin (2 \beta)$ and other $b \rightarrow u$ processes than in the past. Additionally, new results in tagged $B^{+} \rightarrow\left(\mu^{+}\right.$or $\left.e^{+}\right) v$ [52], $B^{+} \rightarrow\left(\mu^{+}\right.$or $\left.e^{+}\right) v \gamma$ [53] and $B^{+} \rightarrow\left(\mu^{+}\right.$or $\left.e^{+}\right) X^{0}$ [54] have been produced by Belle in anticipation of the enormous statistical power that Belle II will bring to bear.

\section{Conclusion}

Looking ahead to the $2018 \mathrm{CKM}$ workshop, more new results are expected in semileptonic $B$ decays on both the theory and experiment fronts. With Belle II on the horizon, further refinements are still needed to be fully ready for the large datasets, particularly from the Lattice, e.g. for $B_{(s)} \rightarrow$ $D_{(s)}^{*}$. In the meantime, LHCb is expected to continue to produce measurements in other $b$ hadron systems such as $B_{s}$, and $b \rightarrow c \tau v$ measurements in a variety of initial and final states. Such further progress is needed and welcome to explore the present puzzles in semileptonic decays, including the inclusive-exclusive branching fraction "gap", the ambiguous tensions in determinations of $\left|V_{u b}\right|$ and the possible enhancement of the semitauonic branching fractions.

\section{Acknowledgements}

We would like to thank the organizers of the CKM conference for their efforts in making this year's workshop so successful. This work is supported in part by the National Science Foundation (NSF), and by the DFG Emmy-Noether Grant No. BE 6075/1-1. 


\section{References}

[1] S. Aoki et al., Eur. Phys. J. C 77 (2017) no.2, 112 doi:10.1140/epjc/s10052-016-4509-7 [arXiv:1607.00299 [hep-lat]].

[2] Y. Amhis et al. [Heavy Flavor Averaging Group], "Average of R(D) and R(D*) for Summer 2016“. http://www.slac.stanford.edu/xorg/hfag/semi/summer16/html/RDsDsstar/RDRDs.html

[3] P. Gambino, K. J. Healey and C. Mondino, Phys. Rev. D 94 (2016) no.1, 014031 doi:10.1103/PhysRevD.94.014031 [arXiv:1604.07598 [hep-ph]].

[4] J. P. Lees et al. [BaBar Collaboration], arXiv:1611.05624 [hep-ex].

[5] A. Abdesselam et al. [Belle Collaboration], arXiv:1702.01521 [hep-ex]

[6] H. Na et al. [HPQCD Collaboration], Phys. Rev. D 92 (2015) no.5, 054510 Erratum: [Phys. Rev. D 93 (2016) no.11, 119906] doi:10.1103/PhysRevD.93.119906, 10.1103/PhysRevD.92.054510 [arXiv:1505.03925 [hep-lat]].

[7] J. M. Flynn, T. Izubuchi, T. Kawanai, C. Lehner, A. Soni, R. S. Van de Water and O. Witzel, Phys. Rev. D 91 (2015) no.7, 074510 doi:10.1103/PhysRevD.91.074510 [arXiv:1501.05373 [hep-lat]].

[8] A. Alberti, P. Gambino, K. J. Healey and S. Nandi, Phys. Rev. Lett. 114 (2015) no.6, 061802 doi:10.1103/PhysRevLett.114.061802 [arXiv:1411.6560 [hep-ph]].

[9] B. Kronenbitter et al. [Belle Collaboration], Phys. Rev. D 92, no. 5, 051102 (2015) doi:10.1103/PhysRevD.92.051102 [arXiv:1503.05613 [hep-ex]].

[10] R. Aaij et al. [LHCb Collaboration], Phys. Rev. Lett. 115, no. 11, 111803 (2015) Addendum: [Phys. Rev. Lett. 115, no. 15, 159901 (2015)] doi:10.1103/PhysRevLett.115.159901, 10.1103/PhysRevLett.115.111803 [arXiv:1506.08614 [hep-ex]].

[11] M. Huschle et al. [Belle Collaboration], Phys. Rev. D 92, no. 7, 072014 (2015) doi:10.1103/PhysRevD.92.072014 [arXiv:1507.03233 [hep-ex]].

[12] Y. Sato et al. [Belle Collaboration], Phys. Rev. D 94, no. 7, 072007 (2016) doi:10.1103/PhysRevD.94.072007 [arXiv:1607.07923 [hep-ex]].

[13] S. Hirose et al. [Belle Collaboration], arXiv:1612.00529 [hep-ex].

[14] I. Caprini, L. Lellouch and M. Neubert, Nucl. Phys. B 530, 153 (1998) doi:10.1016/S0550-3213(98)00350-2 [hep-ph/9712417].

[15] C. J. Monahan, H. Na, C. M. Bouchard, G. P. Lepage and J. Shigemitsu, arXiv:1611.09667 [hep-lat].

[16] J. Harrison, C. Davies and M. Wingate, arXiv:1612.06716 [hep-lat].

[17] R. Glattauer et al. [Belle Collaboration], Phys. Rev. D 93 (2016) no.3, 032006 doi:10.1103/PhysRevD.93.032006 [arXiv:1510.03657 [hep-ex]].

[18] C. G. Boyd, B. Grinstein and R. F. Lebed, Phys. Rev. Lett. 74, 4603 (1995) doi:10.1103/PhysRevLett.74.4603 [hep-ph/9412324].

[19] D. Bigi and P. Gambino, Phys. Rev. D 94, no. 9, 094008 (2016) doi:10.1103/PhysRevD.94.094008 [arXiv:1606.08030 [hep-ph]].

[20] F. U. Bernlochner, Z. Ligeti, M. Papucci and D. J. Robinson, arXiv:1703.05330 [hep-ph].

[21] D. Bigi, P. Gambino and S. Schacht, arXiv:1703.06124 [hep-ph]. 
[22] B. Grinstein and A. Kobach, arXiv:1703.08170 [hep-ph].

[23] C. Oswald et al. [Belle Collaboration], Phys. Rev. D 92 (2015) no.7, 072013 doi:10.1103/PhysRevD.92.072013 [arXiv:1504.02004 [hep-ex]].

[24] B. Colquhoun et al. [LATTICE-HPQCD Collaboration], arXiv:1611.01987 [hep-lat].

[25] S. Fajfer, J. F. Kamenik and I. Nišandžić, Phys. Rev. D 85, 094025 (2012) doi:10.1103/PhysRevD.85.094025 [arXiv:1203.2654 [hep-ph]].

[26] J. A. Bailey et al. [MILC Collaboration], Phys. Rev. D 92, no. 3, 034506 (2015) doi:10.1103/PhysRevD.92.034506 [arXiv:1503.07237 [hep-lat]].

[27] J. P. Lees et al. [BaBar Collaboration], Phys. Rev. D 88, no. 7, 072012 (2013) doi:10.1103/PhysRevD.88.072012 [arXiv:1303.0571 [hep-ex]].

[28] A. Abdesselam et al., arXiv:1608.06391 [hep-ex].

[29] D. Bečirević, S. Fajfer, I. Nišandžić and A. Tayduganov, arXiv:1602.03030 [hep-ph].

[30] I. Doršner, S. Fajfer, A. Greljo, J. F. Kamenik and N. Košnik, Phys. Rept. 641 (2016) 1 doi:10.1016/j.physrep.2016.06.001 [arXiv:1603.04993 [hep-ph]].

[31] D. Bečirević, S. Fajfer, N. Košnik and O. Sumensari, Phys. Rev. D 94 (2016) no.11, 115021 doi:10.1103/PhysRevD.94.115021 [arXiv:1608.08501 [hep-ph]].

[32] S. Sahoo, R. Mohanta and A. K. Giri, Phys. Rev. D 95, no. 3, 035027 (2017) doi:10.1103/PhysRevD.95.035027 [arXiv:1609.04367 [hep-ph]].

[33] J. Selvaganapathy, P. K. Das and P. Konar, Phys. Rev. D 93, no. 11, 116003 (2016) doi:10.1103/PhysRevD.93.116003 [arXiv:1602.02997 [hep-ph]].

[34] Y. Sakaki, M. Tanaka, A. Tayduganov and R. Watanabe, Phys. Rev. D 91 (2015) no.11, 114028 doi:10.1103/PhysRevD.91.114028 [arXiv:1412.3761 [hep-ph]].

[35] A. Bharucha, JHEP 1205 (2012) 092 doi:10.1007/JHEP05(2012)092 [arXiv:1203.1359 [hep-ph]].

[36] I. Sentitemsu Imsong, A. Khodjamirian, T. Mannel and D. van Dyk, JHEP 1502 (2015) 126 doi:10.1007/JHEP02(2015)126 [arXiv:1409.7816 [hep-ph]].

[37] A. Bharucha, D. M. Straub and R. Zwicky, JHEP 1608 (2016) 098 doi:10.1007/JHEP08(2016)098 [arXiv:1503.05534 [hep-ph]].

[38] R. Aaij et al. [LHCb Collaboration], Nature Phys. 11 (2015) 743 doi:10.1038/nphys3415 [arXiv:1504.01568 [hep-ex]].

[39] W. Detmold, C. Lehner and S. Meinel, Phys. Rev. D 92 (2015) no.3, 034503 doi:10.1103/PhysRevD.92.034503 [arXiv:1503.01421 [hep-lat]].

[40] H. Ha et al. [Belle Collaboration], Phys. Rev. D 83 (2011) 071101 doi:10.1103/PhysRevD.83.071101 [arXiv:1012.0090 [hep-ex]].

[41] P. del Amo Sanchez et al. [BaBar Collaboration], Phys. Rev. D 83 (2011) 032007 doi:10.1103/PhysRevD.83.032007 [arXiv:1005.3288 [hep-ex]].

[42] J. P. Lees et al. [BaBar Collaboration], Phys. Rev. D 86 (2012) 092004 doi:10.1103/PhysRevD.86.092004 [arXiv:1208.1253 [hep-ex]].

[43] A. Sibidanov et al. [Belle Collaboration], Phys. Rev. D 88 (2013) no.3, 032005 doi:10.1103/PhysRevD.88.032005 [arXiv:1306.2781 [hep-ex]]. 
[44] J. A. Bailey et al. [Fermilab Lattice and MILC Collaborations], Phys. Rev. D 92 (2015) no.1, 014024 doi:10.1103/PhysRevD.92.014024 [arXiv:1503.07839 [hep-lat]].

[45] P. Gambino, K. J. Healey and S. Turczyk, Phys. Lett. B 763 (2016) 60 doi:10.1016/j.physletb.2016.10.023 [arXiv:1606.06174 [hep-ph]].

[46] S. W. Bosch, B. O. Lange, M. Neubert and G. Paz, Nucl. Phys. B 699, 335 (2004) doi:10.1016/j.nuclphysb.2004.07.041 [hep-ph/0402094].

[47] P. Gambino, P. Giordano, G. Ossola and N. Uraltsev, JHEP 0710 (2007) 058 doi:10.1088/1126-6708/2007/10/058 [arXiv:0707.2493 [hep-ph]].

[48] J. R. Andersen and E. Gardi, JHEP 0701 (2007) 029 doi:10.1088/1126-6708/2007/01/029 [hep-ph/0609250].

[49] F. U. Bernlochner et al. [SIMBA Collaboration], PoS ICHEP 2012 (2013) 370 [arXiv:1303.0958 [hep-ph]].

[50] A. Gunawardana and G. Paz, arXiv:1702.08904 [hep-ph].

[51] I. Adachi et al. [Belle Collaboration], Phys. Rev. Lett. 110, no. 13, 131801 (2013) doi:10.1103/PhysRevLett.110.131801 [arXiv:1208.4678 [hep-ex]].

[52] Y. Yook et al. [Belle Collaboration], Phys. Rev. D 91, no. 5, 052016 (2015) doi:10.1103/PhysRevD.91.052016 [arXiv:1406.6356 [hep-ex]].

[53] A. Heller et al. [Belle Collaboration], Phys. Rev. D 91, no. 11, 112009 (2015) doi:10.1103/PhysRevD.91.112009 [arXiv:1504.05831 [hep-ex]].

[54] C. S. Park et al. [Belle Collaboration], Phys. Rev. D 94, no. 1, 012003 (2016) doi:10.1103/PhysRevD.94.012003 [arXiv:1605.04430 [hep-ex]]. 